\title{
Prediction of haplotypes for ungenotyped animals and its effect on marker-assisted breeding value estimation
}

\author{
Han A Mulder*, Mario PL Calus and Roel F Veerkamp
}

\begin{abstract}
Background: In livestock populations, missing genotypes on a large proportion of animals are a major problem to implement the estimation of marker-assisted breeding values using haplotypes. The objective of this article is to develop a method to predict haplotypes of animals that are not genotyped using mixed model equations and to investigate the effect of using these predicted haplotypes on the accuracy of marker-assisted breeding value estimation.
\end{abstract}

Methods: For genotyped animals, haplotypes were determined and for each animal the number of haplotype copies (nhc) was counted, i.e. 0, 1 or 2 copies. In a mixed model framework, nhc for each haplotype were predicted for ungenotyped animals as well as for genotyped animals using the additive genetic relationship matrix. The heritability of nhc was assumed to be 0.99, allowing for minor genotyping and haplotyping errors. The predicted nhc were subsequently used in marker-assisted breeding value estimation by applying random regression on these covariables. To evaluate the method, a population was simulated with one additive QTL and an additive polygenic genetic effect. The QTL was located in the middle of a haplotype based on SNP-markers.

Results: The accuracy of predicted haplotype copies for ungenotyped animals ranged between 0.59 and 0.64 depending on haplotype length. Because powerful BLUP-software was used, the method was computationally very efficient. The accuracy of total EBV increased for genotyped animals when marker-assisted breeding value estimation was compared with conventional breeding value estimation, but for ungenotyped animals the increase was marginal unless the heritability was smaller than 0.1. Haplotypes based on four markers yielded the highest accuracies and when only the nearest left marker was used, it yielded the lowest accuracy. The accuracy increased with increasing marker density. Accuracy of the total EBV approached that of gene-assisted BLUP when 4-marker haplotypes were used with a distance of $0.1 \mathrm{cM}$ between the markers.

Conclusions: The proposed method is computationally very efficient and suitable for marker-assisted breeding value estimation in large livestock populations including effects of a number of known QTL. Marker-assisted breeding value estimation using predicted haplotypes increases accuracy especially for traits with low heritability.

\section{Background}

In livestock, many QTL regions have been identified for quantitative traits [1]. In some cases, fine mapping has also led to the detection of causative mutations, e.g. DGAT1 in dairy cattle for milk yield and milk composition $[2,3]$ and IGF2 in pigs for body weight [4]. In breeding programs these QTL-regions can be utilized in

\footnotetext{
* Correspondence: herman.mulder@wur.nl

1 Animal Breeding and Genomics Centre, Wageningen UR Livestock Research, PO Box 65, 8200 AB Lelystad, The Netherlands

Full list of author information is available at the end of the article
}

marker-assisted selection (MAS). Three types of markers can be used: markers in linkage equilibrium with the QTL (LE-MAS), markers in linkage disequilibrium with the QTL (LD-MAS) and the causative mutation itself as in gene-assisted selection (GAS). GAS leads to the highest genetic gain, because no recombination exists between the marker and QTL [5]. However, identifying the gene is not easy and is resource demanding [1]. The amount of QTL variation explained by markers in LD-MAS can be increased by increasing the marker density and thereby 
increasing the LD between markers and QTL. Alternatively, combining alleles of different marker loci into haplotypes is expected to increase the proportion of captured QTL variance as well. Based on data of a whole genome scan with 9323 SNP-markers in Angus cattle, Hayes et al. [6] have reported that 4 and 6-marker haplotypes increased the accuracy of MAS more than the single marker in highest LD with the QTL. However, 2-marker haplotypes performed worse than the best marker.

One of the challenges when applying MAS in livestock populations is that often a large part of the population is not genotyped, i.e. some animals have only phenotypes, some have only genotypes and others have both genotypes and phenotypes. Several methods have been proposed to overcome these differences. For LE-MAS, one would like to apply a method that uses identity-by-descent (IBD) information of haplotypes to properly account for relationships between haplotypes of related animals and to account for phase differences between markers and QTL in different families [7]. Creation of inverse IBD-matrices is, however, very time consuming [8]. With high-density SNP-chips, LD-MAS can be applied without having to use IBD-matrices. With LD-MAS, either flanking markers or identical-by-state haplotypes (IBS) can be used in markerassisted breeding value estimation. When using flanking markers in MAS, genotype probabilities could be calculated with iterative peeling methods [9-13] but these are time consuming. Gengler et al. $[14,15]$ have proposed a straightforward and quick method to predict genotype probabilities and gene contents for bi-allelic markers using a mixed model methodology, where gene content is the number of positive (negative) alleles (i.e. 2, 1, 0 for AA, Aa, aa). For ungenotyped animals, the accuracy of predicted gene contents is similar whether mixed model equations or single-marker iterative peeling are used [8,14]. Gengler et al. [14] suggested that the method can also be applied in the case of multi-allelic markers. Multi-marker IBS haplotypes can be considered as a special form of multi-allelic markers, making the mixed model methodology a candidate method to predict haplotypes for ungenotyped animals.

The objective of this article is to develop a method to predict haplotypes of animals that are not genotyped using mixed model equations and to investigate the effect of using those predicted haplotypes on the accuracy of marker-assisted breeding value estimation. The method is evaluated using Monte Carlo simulation, varying haplotype length, heritability of the trait and distance between the markers. The method is compared to geneassisted and conventional breeding value estimation, which yield, respectively, the upper and lower limit of accuracy.

\section{Methods}

\section{Prediction of haplotypes with missing genotypes}

Consider a situation where a QTL-region is mapped for a trait, without having identified the causative mutation and where some animals in the population are genotyped for SNP-markers in that region, but most of them are not genotyped, which is very common in animal breeding populations. In this study we would like to use IBS-haplotypes in marker-assisted breeding value estimation. When the haplotype is based on the single SNP-marker closest to the QTL, the method of Gengler et al. [14,15] can be used to predict the missing 'gene content', the number of A-alleles, if there are A and a-alleles. The method of Gengler et al. $[14,15]$ uses the additive genetic relationship matrix in a mixed model setting to predict the gene contents of those animals not genotyped based on genotyped relatives. This method can not be applied directly for haplotypes based on multiple markers, because discrete haplotypes can not be directly constructed based on predicted continuous gene contents of SNP-markers for ungenotyped animals. However, this procedure can be easily modified to apply to a situation with haplotypes based on multiple markers. Consider that haplotypes are based on two bi-allelic markers, one on each side of the QTL. There are four possible haplotypes. For every genotyped animal, one can infer how many copies it carries for each haplotype $(n h c=$ number of haplotype copies), which is 0,1 or 2 (see Table 1 for a small example). This is in essence the same as the 'gene content' for a bi-allelic locus and the same mixed model methodology with the additive genetic relationship matrix can be applied to predict the $n h c$ for each haplotype for the ungenotyped animals. In the case of $n$ haplotypes this can be modeled as:

Table 1: Example with four animals with the number of haplotype copies for two SNP-marker haplotypes

Number of haplotype copies (nhc)

\begin{tabular}{ccccccc}
\cline { 3 - 6 } Animal & Haplotype 1 & Haplotype 2 & Hap1 (11) & Hap2 (12) & Hap3 (21) & Hap4 (22) \\
\hline 1 & 11 & 11 & 2 & 0 & 0 & 0 \\
2 & 11 & 12 & 1 & 1 & 0 & 0 \\
3 & 11 & 21 & 1 & 0 & 0 & 0 \\
4 & 11 & 22 & 1 & 0 & 0 & 1 \\
\hline
\end{tabular}




$$
n h c_{i}=\mu_{n h c_{i}}+d_{i}+e_{n h c_{i}}
$$

where $n h c_{i}$ is the number of copies of haplotype $i$ (which is 0,1 or 2 effectively), $\mu_{n h c_{i}}$ is the population mean number of copies of haplotype $i, d_{i}$ is the EBV for $n h c_{i}$ and $e_{n h c_{i}}$ is the residual of $n h c_{i}$. Although $\sum_{i=1}^{n} n h c_{i}=2$ for each animal, it is assumed that the haplotypes are independent from each other; therefore $n$ univariate mixed model analyses can be performed. Analogous to gene contents for a bi-allelic locus [14], this can be formulated in mixed model matrix notation as:

$$
\left[\begin{array}{cc}
\mathbf{1}^{\prime} \mathbf{1} & \mathbf{1}^{\prime} \mathbf{M} \\
\mathbf{M}^{\prime} \mathbf{1} & \mathbf{M}^{\prime} \mathbf{M}+\lambda \mathbf{A}^{-1}
\end{array}\right]\left[\begin{array}{c}
\mu_{n h c} \\
\mathbf{d}_{y} \\
\mathbf{d}_{x}
\end{array}\right]=\left[\begin{array}{c}
\mathbf{1}^{\prime} \mathbf{n h} \mathbf{c}_{\mathbf{y}} \\
\mathbf{M}^{\prime} \mathbf{n h} \mathbf{c}_{\mathbf{y}}
\end{array}\right]
$$

where $\mathbf{1}$ is a vector of ones, $\mathbf{M}$ is a design matrix linking d with $\mathbf{n h c}_{y}, \mathbf{A}^{-1}$ is the inverse additive genetic relationship matrix, $\lambda$ is the variance ratio of residual variance and additive genetic variance for $n h c$ allowing for a small proportion of genotyping and haplotyping errors or recombination $\lambda=\sigma_{e_{n h c}}^{2} / \sigma_{a_{n h c}}^{2}=0.01 / 0.99, \mathbf{d}$ is a vector with the EBV for $n h c$ with $\mathbf{d}_{y}$ for genotyped animals and $\mathbf{d}_{x}$ for ungenotyped animals, nhc $\mathbf{c}_{y}$ is a vector with observed $n h c$ of genotyped animals and is set to missing for ungenotyped animals. The heritability assumed for $n h c$ is 0.99 . Basically, with no genotyping or haplotyping errors, $u_{n h c_{i}}+d_{i}$ (the predicted $n h c$ ) should be equal to the phenotype (the true $n h c$ ) for genotyped animals, implying a heritability of 1.0. In the case of haplotypes, recombinant haplotypes can be transmitted from one parent to its offspring. In such a case, the recombinant haplotype can not be fully explained in the model by the haplotypes of the parent. This decreases the parent-offspring regression, i.e. decreasing the heritability. Here we set the heritability to 0.99 to allow for some small proportions of genotyping and haplotyping errors and recombi- nation. Preliminary analysis showed no effect when the heritability was changed to 0.95 .

\section{Marker-assisted breeding value estimation using predicted} haplotypes

To include the effects of the haplotypes to perform marker-assisted breeding value estimation using best linear unbiased prediction (MABLUP), these $n h c$ can be used as covariables in random regression, where inclusion as a random effect is preferred so that effects will be regressed towards zero when there is hardly any phenotypic information, e.g. a certain haplotype appears only in one animal with a phenotypic record. Assuming no other systematic environmental effects, the model is as follows:

$$
y=\mu+u_{p o l}+\sum_{i}^{n}\left(n \hat{h} c_{i} \times h_{i}\right)+e
$$

where $y$ is the phenotype, $\mu$ is the overall mean and modeled as a fixed effect, $u_{p o l}$ is the random polygenic EBV, $n \hat{h} c_{i}=\mu_{n h c_{i}}+d_{i}$, which is the predicted number of copies of haplotype $i, h_{i}$ is the random regression coefficient for haplotype $i$ and $e$ is the residual. In matrix notation the model can be summarized as:

$$
\left[\begin{array}{ccc}
\mathbf{X}^{\prime} \mathbf{X} & \mathbf{X}^{\prime} \mathbf{Z} & \mathbf{X}^{\prime} \mathbf{W} \\
\mathbf{Z}^{\prime} \mathbf{X} & \mathbf{Z}^{\prime} \mathbf{Z}+\lambda_{p o l} \mathbf{A}^{-\mathbf{1}} & \mathbf{Z}^{\prime} \mathbf{W} \\
\mathbf{W}^{\prime} \mathbf{X} & \mathbf{W}^{\prime} \mathbf{Z} & \mathbf{W}^{\prime} \mathbf{W}+\lambda_{h}
\end{array}\right]\left[\begin{array}{c}
\mathbf{b} \\
\mathbf{u}_{p o l} \\
\mathbf{h}_{i}
\end{array}\right]=\left[\begin{array}{c}
\mathbf{X}^{\prime} \mathbf{y} \\
\mathbf{Z}^{\prime} \mathbf{y} \\
\mathbf{w}_{i}^{\prime} \mathbf{y}
\end{array}\right]
$$

where $\mathbf{X}$ and $\mathbf{Z}$ are the design matrices for fixed effects and polygenic breeding values, respectively, the matrix $\mathbf{W}$ contains the $n \hat{h} c$ for all haplotypes, $\lambda_{p o l}$ and $\lambda_{h}$ are respectively the variance ratios for the polygenic breeding values and the random regression on $n \hat{h} c_{i}$, $\mathbf{b}$ is the vector with solutions for fixed effects (in this case only the mean), $\mathbf{u}_{p o l}$ is the vector with $u_{p o l}$ and $\mathbf{h}_{i}$ is the vector with $h_{i}$. The variance of $h_{i}$ is $\sigma_{h}^{2}=0.5 \sigma_{A_{q t l}}^{2}$ (see Appendix for derivation), where $\sigma_{A_{q t l}}^{2}$ is the additive genetic QTL-variance, and the variance of $u_{p o l}$ is $\sigma_{u_{p o l}}^{2}=\sigma_{A_{p o l}}^{2}$, where $\sigma_{A_{p o l}}^{2}$ is the additive genetic variance due to the polygenic effect. Equations (3) and (4) can be considered as a gener- 
alization of the method by Gengler et al. $[14,15]$ to multiallelic markers and haplotypes.

\section{Evaluation of method Simulation}

Monte Carlo simulation was used to evaluate the method. The simulation scheme represented a nested full-sib halfsib design (multiple offspring per mating and dam nested within sire) with discrete generations which is common in commercial animal breeding programs. The simulation scheme was identical to that reported in Mulder et al. [8]. One trait was simulated with additive genetic effects of one bi-allelic QTL $A_{q t}$, a polygenic additive genetic effect $A_{p o l}$ and a residual effect $e\left(P=A_{q t l}+A_{p o l}\right.$ $+e)$. All animals had phenotypic records. Because the method of MABLUP relies on linkage disequilibrium (LD) between markers and QTL, first, 100 generations of random mating were performed prior to the data collection scheme (generation 101 - 105).

In the first 100 generations, 50 sires and 50 dams were randomly mated each generation. The QTL and 20 biallelic markers were placed on one $1 \mathrm{M}$ long chromosome. The QTL was placed in the middle of the chromosome and the markers were equally spaced, their distance varying from 0.1 to $5 \mathrm{cM}$. The QTL was in the middle of the marker bracket between marker 10 and 11. In the founder generation, all markers and the QTL were in linkage equilibrium and had a fixed allele frequency of 0.5. The QTL-variance $\sigma_{A_{q t l}}^{2}$ was set to $15 \%$ of the total genetic variance, when the allele frequency is 0.5 . The allele substitution effect was set to $a=\sqrt{\sigma_{A_{q t l}}^{2} / 2 p q}$, assuming that the allele frequencies $p$ and $q$ are 0.5 , which is the case in the founder generation. Recombination rates were calculated using Haldane's mapping function [16]. During these 100 generations, some markers or the QTL became fixed due to drift.

After establishing LD, from generation 101 onwards and for each generation 50 sires and 250 dams were selected based on conventional BLUP-EBV (Equation (3) without haplotype effects) and randomly mated to produce 2,000 offspring. Each sire was mated to five dams and each dam produced four male and four female offspring, resulting in that each sire had 40 half-sib offspring, five full-sib groups of eight full-sibs. A total of five generations of phenotypic data (generation 101 - 105) were created and used in breeding value estimation (10,000 animals in total). The animals of generation 101 served as base generation in the pedigree. The generations 102 - 104 were used to create linkage disequilibrium due to selection [17].

In generation 101, simulated polygenic effects were sampled from $N\left(0, \sigma_{A_{p o l}}^{2}\right)$, where $\sigma_{A_{p o l}}^{2}$ is the polygenic genetic variance. In subsequent generations polygenic effects were sampled from $N\left(0.5 A_{p o l, s}+0.5 A_{p o l, d}, 0.5\right.$ $\left.\sigma_{A_{p o l}}^{2}\left(1-f_{p}\right)\right)$, where $f_{p}$ is the average inbreeding coefficient of the parents. Inbreeding coefficients were calculated using the Meuwissen and Luo [18] algorithm. Residual effects were sampled from $N\left(0, \sigma_{e}^{2}\right)$, where $\sigma_{e}^{2}$ is the residual variance.

The overall heritability was set to $0.03,0.10$ or 0.30 , while the QTL explained 15\% of the total genetic variance when the allele frequency was 0.5 as it was in the founder generation. The phenotypic variance was 1.0 in all situations when the allele frequency of the QTL was 0.5. The realized variance of the QTL was lower due to deviations of the allele frequency from 0.5 and re-estimated in generation 101. Results were based on 200 effective replicates after discarding the replicates with minor allele frequency of the QTL in the last generation (generation 105) less than 0.05. Averaged over all effective replicates, the average allele frequency of the negative QTL-allele was 0.63 in generation 101 before selection started and deviated from 0.5 , because in replicates with allele frequencies closer to 0, the QTL was more likely to become fixed in generations 101-105 due to selection. The used parameter values are listed in Table 2.

Haplotype methods used for marker-assisted breeding value estimation

In this study we used three types of haplotypes: 1) the closest neighboring left marker of the QTL is used as a single-marker haplotype (NM), 2) both flanking markers closest to the QTL-locus are used to form a 2-marker haplotype (HAP2) and 3) on both sides the two markers closest to the QTL are used to form a 4-marker haplotype (HAP4). In the case of NM, Equation (3) and (4) reduced to the method by Gengler et al. [14,15] with the difference that in this case it was not the causative mutation, but a linked marker. In addition, $\sigma_{h}^{2}=\alpha^{2}$, where $\alpha$ is the allele substitution effect (see equation A1 in the Appen- 
Table 2: Parameter values for simulation

\begin{tabular}{|c|c|c|}
\hline Parameter & Default value & Alternative values \\
\hline Number of sires per generation & 50 & \\
\hline Number of dams per generation & 250 & \\
\hline Total number of animals & 10,000 & \\
\hline Number of progeny per dam & 8 & \\
\hline Number of generations & 5 & \\
\hline Heritability & 0.3 & 0.03 and 0.10 \\
\hline $\begin{array}{l}\text { Proportion of genetic variance explained } \\
\text { by QTL }\end{array}$ & 0.15 & \\
\hline Number of markers simulated & 20 & \\
\hline Distance between markers & $0.1 \mathrm{cM}$ & $0.5,1.0,2.0,5.0 \mathrm{cM}$ \\
\hline Number of markers used & 10 & \\
\hline Number of replicates & 200 & \\
\hline
\end{tabular}

dix), because we modeled only one SNP marker allele. The markers chosen to form haplotypes had minor allele frequencies of at least $5 \%$ in generation 105. Haplotypes were known from the simulation and thus, phasing was not needed.

\section{Genotyping and breeding value estimation}

In generation 105, the breeding program starts with MABLUP according to Equation (3) and (4) using the three different haplotype methods. We simulated three genotyping scenarios: (1) only sires and males in the last generation are genotyped and (default) (2) all males are genotyped and (3) all animals are genotyped. In scenario 1 and 2, females are not genotyped. In addition to MABLUP, gene-assisted BLUP (GABLUP) and conventional BLUP (CONBLUP) are also performed for comparison. For GABLUP, it is assumed that all animals are genotyped for the QTL. For GABLUP the model is equal to Equation (3), with the difference that the true gene content is used as $n h c$ and the variance is the same as for NM. For CONBLUP, Equation (3) is used without regression on $n h c$ and the variance of the additive genetic effect is set to $\sigma_{u}^{2}=\sigma_{A_{p o l}}^{2}+\sigma_{A_{q t l}}^{2}$. For all evaluations, mixed model equations were solved using MiX99, which makes use of the preconditioned conjugate gradient algorithm [19]. The mixed model equations were considered converged when the relative difference between the left-hand and right-hand sides of the mixed model equations was smaller than $1.0 * 10^{-10}$

Accuracies were calculated as correlations between estimated and true breeding values. The QTL-EBV was calculated as $\sum_{i}^{n}\left(n \hat{h} c_{i} \times h_{i}\right)$ for each animal. The total EBV was calculated as the sum of the QTL-EBV and the polygenic EBV. Accuracies of MABLUP were compared to those of GABLUP and CONBLUP. The accuracies of GABLUP and CONBLUP can be considered as the upper and lower limits for the MABLUP accuracy. In addition, regressions of true breeding values on estimated breeding values were calculated to get an idea of the over- (regression coefficient $<1.0$ ) or underestimation (regression coefficient $>1.0$ ) of the variance of EBV. Bias of estimated breeding values was calculated as estimated breeding values minus true breeding values. In addition, accuracies of $n \hat{h} c$ were calculated as correlations between estimated and true $n h c$ and regressions of true on estimated $n h c$ were calculated.

\section{Proportion of QTL-variance explained by the haplotypes}

The proportion of QTL-variance explained by the three different haplotypes NM, HAP2 and HAP4 was calculated to assess whether using IBS-haplotypes was suitable. The proportion of QTL-variance explained by the 
Table 3: Summary statistics of predicted number of haplotype copies for ungenotyped animals

\begin{tabular}{|c|c|c|c|c|}
\hline Haplotype method & True nhc & Mean & SD & MSE \\
\hline \multirow[t]{3}{*}{ NM } & 0 & 0.59 & 0.08 & 0.54 \\
\hline & 1 & 0.99 & 0.09 & 0.20 \\
\hline & 2 & 1.43 & 0.08 & 0.52 \\
\hline \multirow[t]{3}{*}{ HAP2 } & 0 & 0.34 & 0.06 & 0.27 \\
\hline & 1 & 0.76 & 0.08 & 0.24 \\
\hline & 2 & 1.24 & 0.08 & 0.75 \\
\hline \multirow[t]{3}{*}{ HAP4 } & 0 & 0.16 & 0.04 & 0.11 \\
\hline & 1 & 0.58 & 0.06 & 0.32 \\
\hline & 2 & 1.13 & 0.08 & 0.90 \\
\hline
\end{tabular}

Mean, standard deviation (SD) and mean square error (MSE) of predicted number of haplotype copies (nhc) for neighboring marker (NM), 2marker haplotype (HAP2) and 4-marker haplotypes (HAP4) for ungenotyped animals in the last generation (females) as a function of true nhc (sires and males in last generation are genotyped; distance between markers is $0.1 \mathrm{cM}$, heritability is 0.30 , the QTL explains $15 \%$ of the genetic variance, results are averages of 200 replicates)

haplotypes is also a measure of linkage disequilibrium between the haplotype and the QTL. For NM, the $r^{2}$ between the marker and the QTL can be calculated as the squared correlation between them [20]. For multi-allelic haplotypes, such as HAP2 and HAP4, $r^{2}$ was calculated according to Equation (2) in Hayes et al. [6], based on an equation for multi-allelic markers by Zhao et al. [21].

\section{Results}

\section{Analysis of haplotypes}

\section{Statistics of predicted number of haplotype copies}

Table 3 shows the mean, standard deviation and mean square error (MSE) for predicted number of haplotype copies $(n h c)$ for ungenotyped animals as a function of the true number of haplotype copies. For all three methods, the predicted $n h c$ increased with the true $n h c$ and a clear distinction was made in $n h c$ between animals carrying the haplotype or not. For genotyped animals the predicted $n h c$ closely resembled the true $n h c$. For ungenotyped animals, the absolute numbers decreased from NM towards HAP4, due to regression to the mean and the mean $n h c$ decreased from NM towards HAP4, albeit the difference between homozygotic carrier and non-carrier is largest for HAP4. As a consequence, the MSE increased with increasing true $n h c$ for HAP2 and HAP4 and for HAP4 more than for HAP2. In general, the mean $n h c$ decreased with the frequency of the haplotype (results not shown).

Table 4 shows the accuracy of predicted $n h c$ and the regression of true $n h c$ on predicted $n h c$ for ungenotyped females. The accuracy decreased from NM towards HAP4, especially for HAP4, due to recombination between genotyped ancestors and ungenotyped offspring. Especially for HAP4, the accuracy decreased when the marker distance increased, which is again due to a higher probability of recombination (results not shown). The regression of true $n h c$ on predicted $n h c$ was approximately 1 for NM and HAP2, but somewhat lower for HAP4, due to the lower accuracy.

Proportion of QTL-variance explained by haplotype

Figure 1 shows the mean proportion of QTL variance $\left(r^{2}\right)$ explained by the haplotype as a function of marker dis-

Table 4: Accuracy and regression coefficients of predicted number of haplotype copies for ungenotyped animals

\begin{tabular}{cccc}
\hline Haplotype method & Accuracy nhc (se) & Regression $\mathbf{~ t r u e ~ n h c ~ o n ~ p r e d i c t e d ~ n h c ~ ( s e ) ~}$ \\
\hline NM & 0.643 & $(0.003)$ & $(0.004)$ \\
HAP2 & 0.630 & $(0.007)$ & $(0.022)$ \\
HAP4 & 0.595 & $(0.012)$ & 0.994 \\
\hline
\end{tabular}

Accuracy of number of haplotype copies ( $n h c)$ and regression of true $n h c$ on predicted $n h c$ for neighboring marker (NM), 2-marker haplotype (HAP2) and 4-marker haplotypes (HAP4) for ungenotyped animals in the last generation (females) (sires and males in last generation are genotyped; distance between markers is $0.1 \mathrm{cM}$, heritability is 0.30 , the QTL explains $15 \%$ of the genetic variance, results are averages of 200 replicates)

${ }^{1}$ Regressions where the variance of the predicted $n h c$ was smaller than 0.0001 were omitted (denominator of regression coefficient) 


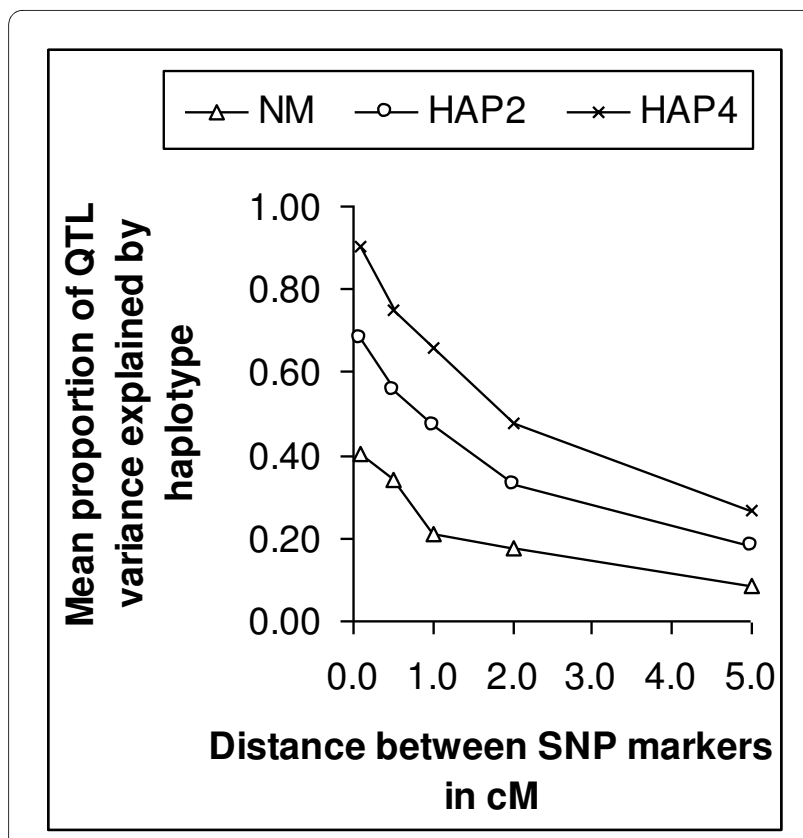

Figure 1 Mean proportion of QTL-variance explained by haplotypes as a function of distance between SNP-markers. Mean proportion of QTL-variance explained by neighboring marker (NM), 2marker haplotype (HAP2) and 4-marker haplotype (HAP4); average of 200 replicates.

tance. For all three methods, $\mathrm{r}^{2}$ decreased with increasing marker distance. The HAP4 method captured most of the QTL variance and NM the least. Figure 2 shows the frequency distribution of $\mathrm{r}^{2}$ values for the three methods at a marker density of $0.1 \mathrm{cM}$. It shows that HAP4 had the highest proportion of replicates with $\mathrm{r}^{2}$ values between 0.90 and 1.00. With NM and HAP2, a substantial proportion of replicates had $\mathrm{r}^{2}$ values below $20 \%$ indicating that the haplotype explained very little QTL-variance.

\section{Accuracy of EBV \\ Effect of genotyping scenario}

Table 5 shows the accuracies of QTL-EBV, polygenic EBV and total EBV for genotyped males and ungenotyped females under different genotyping scenarios with the three methods of MABLUP when the marker distance was $0.1 \mathrm{cM}$. The accuracy of polygenic and total EBV hardly changed when the number of genotyped animals increased. The accuracy of QTL-EBV increased only slightly with an increasing number of genotyped animals. This means that the use of predicted haplotypes in MABLUP did not negatively affect the accuracy of EBV. Because of the small differences in accuracy, in the rest of the article we only show results under the scenario where sires and males in the last generation were genotyped.

\section{Effect of marker density}

Figure 3 shows the accuracy of QTL-EBV (panel A and B) and total EBV (Panel $C$ and D) for genotyped males (panel $\mathrm{A}$ and $\mathrm{C}$ ) and ungenotyped females (panel $\mathrm{B}$ and

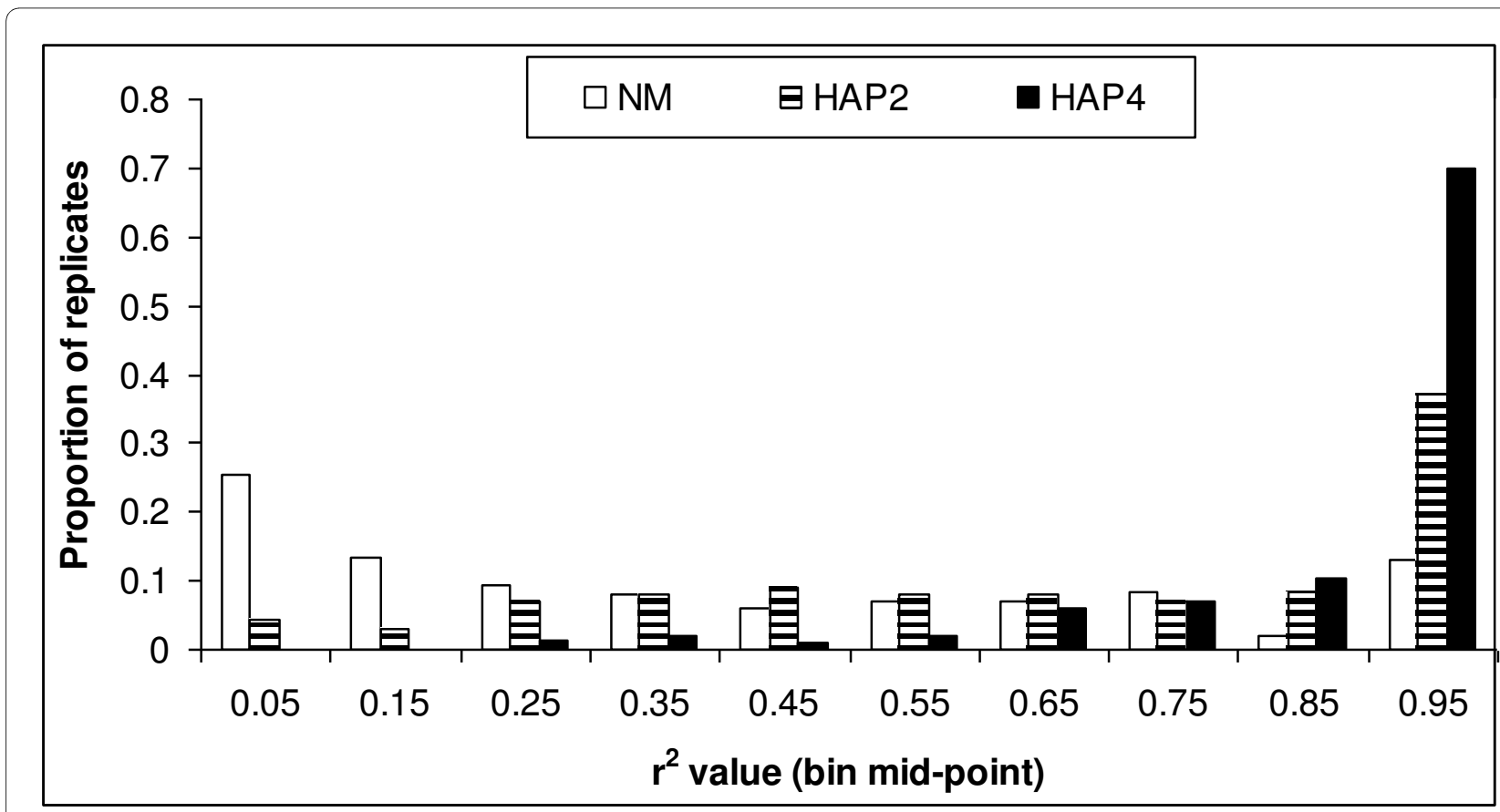

Figure 2 Frequency distribution of QTL-variance explained by haplotypes. Proportion of replicates per 0.1-bin class of proportion of QTL variance $\left(r^{2}\right)$ explained by neighboring marker (NM), 2-marker haplotype (HAP2) and 4-marker haplotype (HAP4); average of 200 replicates; sires and males in last generation are genotyped; distance between markers is $0.1 \mathrm{cM}$. 
Table 5: Accuracy of EBV for genotyped males and ungenotyped females in different genotyping scenarios

\begin{tabular}{|c|c|c|c|c|c|c|c|}
\hline \multirow[b]{2}{*}{ EBV } & \multirow[b]{2}{*}{ Scenario $^{2}$} & \multicolumn{3}{|c|}{ Genotyped } & \multicolumn{3}{|c|}{ Ungenotyped } \\
\hline & & NM & HAP2 & HAP4 & NM & HAP2 & HAP4 \\
\hline \multirow[t]{3}{*}{ QTL } & $\begin{array}{c}\text { sires }+ \\
\text { males last }\end{array}$ & 0.534 & 0.775 & 0.912 & 0.336 & 0.491 & 0.580 \\
\hline & $\begin{array}{l}\text { all males } \\
\text { genotyped }\end{array}$ & 0.534 & 0.774 & 0.926 & 0.337 & 0.493 & 0.591 \\
\hline & $\begin{array}{c}\text { all } \\
\text { genotyped }\end{array}$ & 0.534 & 0.776 & 0.932 & & & \\
\hline \multirow[t]{3}{*}{ Polygenic } & $\begin{array}{c}\text { only sires + } \\
\text { males last }\end{array}$ & 0.567 & 0.576 & 0.583 & 0.566 & 0.575 & 0.582 \\
\hline & $\begin{array}{l}\text { all males } \\
\text { genotyped }\end{array}$ & 0.567 & 0.577 & 0.584 & 0.566 & 0.576 & 0.583 \\
\hline & $\begin{array}{c}\text { all } \\
\text { genotyped }\end{array}$ & 0.567 & 0.578 & 0.586 & & & \\
\hline \multirow[t]{3}{*}{ Total } & $\begin{array}{c}\text { only sires }+ \\
\text { males last }\end{array}$ & 0.605 & 0.616 & 0.622 & 0.595 & 0.596 & 0.596 \\
\hline & $\begin{array}{l}\text { all males } \\
\text { genotyped }\end{array}$ & 0.605 & 0.616 & 0.624 & 0.595 & 0.596 & 0.596 \\
\hline & $\begin{array}{c}\text { all } \\
\text { genotyped }\end{array}$ & 0.606 & 0.617 & 0.625 & & & \\
\hline \multicolumn{8}{|c|}{$\begin{array}{l}\text { Accuracies }{ }^{1} \text { of QTL-EBV, polygenic EBV and total EBV for different genotyping scenarios for marker-assisted BLUP with neighboring marker } \\
\text { (NM), 2-marker haplotype (HAP2) and } 4 \text {-marker haplotypes (HAP4) (distance between markers is } 0.1 \mathrm{cM} \text {, heritability is } 0.30 \text {, the QTL explains } \\
15 \% \text { of the genetic variance, results are averages of } 200 \text { replicates) } \\
\text { 'Standard errors were between } 0.005 \text { and } 0.021 \text { for QTL_EBV, between } 0.002 \text { and } 0.003 \text { for polygenic and total EBV; }{ }^{2} \text { in the first scenario sires } \\
\text { from generation } 101-104 \text { and males in generation } 105 \text { were genotyped ( } 1,200 \text { genotyped animals); in scenario } 2 \text { all males were genotyped } \\
\text { (5,000 genotyped animals) and in the last scenario all animals are genotyped ( } 10,000 \text { genotypes) }\end{array}$} \\
\hline
\end{tabular}

D) as a function of marker distance using three different haplotype methods for MABLUP or using CONBLUP or GABLUP when all animals were genotyped. For genotyped males (Figure 3A) the accuracy of the QTL-EBV was between 0.22 and 0.90 for NM, HAP2 and HAP4 and 1.0 for GABLUP. Among the three haplotype methods, HAP4 had the highest accuracy and NM the lowest. The accuracy decreased with increasing marker distance and more rapidly for HAP4 than for NM, due to a decreasing proportion of QTL variance explained by the haplotypes (Figure 1). For ungenotyped females (Figure 3B), the accuracy of the QTL-EBV was much lower than for genotyped males, between 0.15 and 0.57 for NM, HAP2 and HAP4, but with the same trends across marker distances as for genotyped animals. The MABLUP methods based on HAP2 and HAP4 were both able to increase substantially the accuracy of the total EBV of genotyped males in comparison to CONBLUP when the distance between the markers was small (Figure 3C). The accuracy of MABLUP with HAP4 approached the accuracy of geneassisted BLUP when the marker distance was $0.1 \mathrm{cM}$ or less. The advantage of MABLUP was negligible when the marker distance was large, e.g. 5 cM. For ungenotyped animals (Figure 3D), the increase in accuracy of total EBV of MABLUP over conventional BLUP was, however, negligible regardless of marker distance.

Although the average accuracy of QTL-EBV was moderate to high for genotyped males when markers were separated by $0.1 \mathrm{cM}$, substantial variation existed between replicates (Figure 4). Especially with NM, the variation between replicates was large and even negative accuracies were obtained, although in a very small proportion of the replicates (5.5\% of replicates). With HAP4, accuracies of QTL-EBV were always positive and in $86.5 \%$ of the replicates larger than 0.80 . With HAP2 this proportion equaled to $60 \%$ and with NM only to $30.5 \%$. The figure clearly shows that HAP4 had not only the highest average accuracy, but also the least variation in accuracy of QTL-EBV.

\section{Effect of heritability}

Table 6 shows the accuracies of QTL-EBV, polygenic EBV and total EBV for genotyped males and ungenotyped females using different values of heritability in the three MABLUP methods when the marker distance was 0.1 

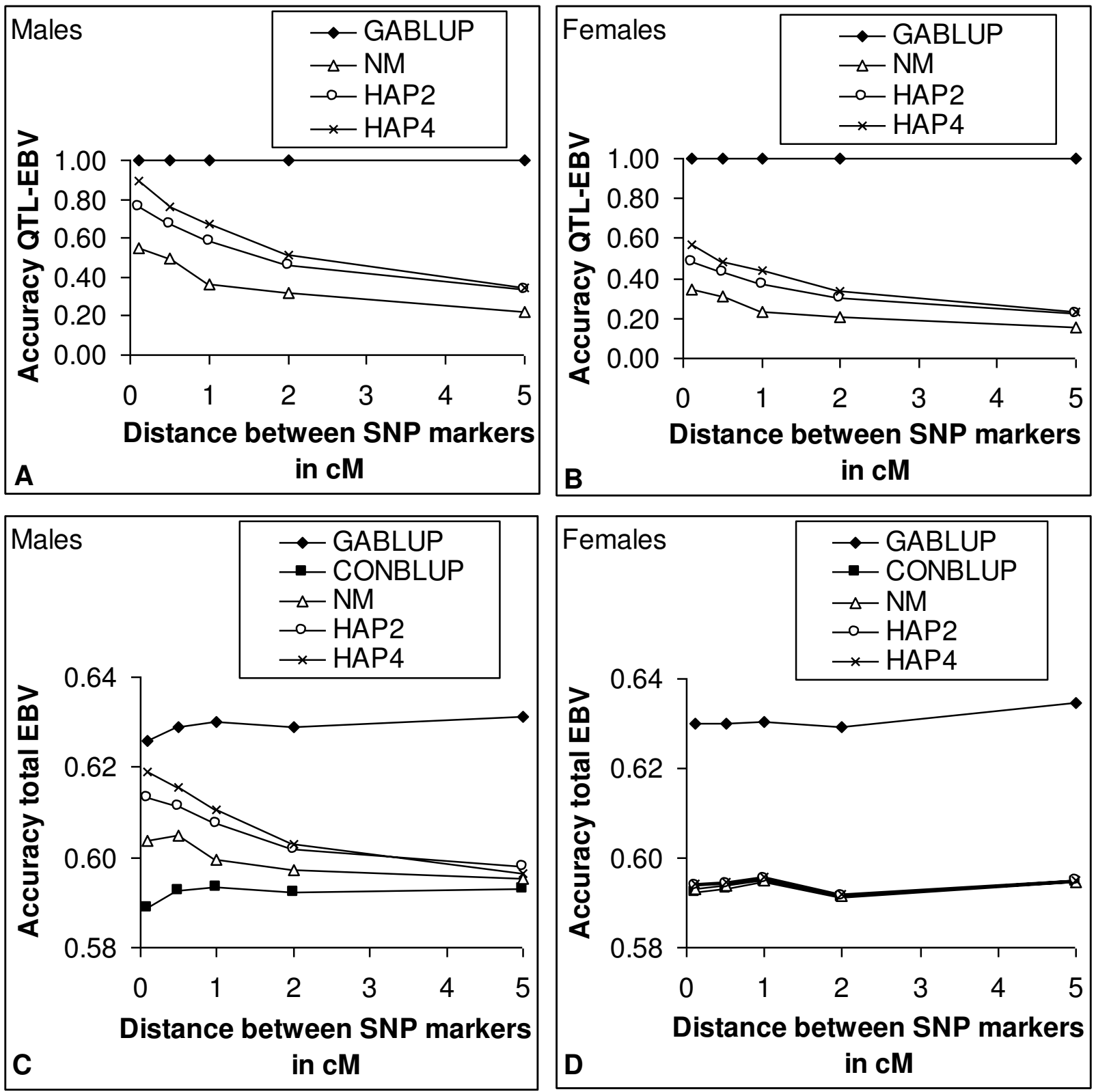

Figure 3 Accuracy of QTL-EBV and total EBV as a function of marker distance for genotyped males and ungenotyped females. Accuracy of QTL-EBV and total EBV for marker-assisted BLUP with neighboring marker (NM), 2-marker haplotype (HAP2) and 4-marker haplotype (HAP4), geneassisted BLUP (GABLUP) when all animals are genotyped and conventional BLUP (CONBLUP); panels A and B: accuracy of QTL-EBV; panels C and D accuracy of total EBV; for MABLUP, sires and males in the last generation were genotyped, the rest was not genotyped, heritability is 0.30 , the QTL explains $15 \%$ of the genetic variance, results are averages of 200 replicates.

cM. The accuracy of QTL-EBV increased with increasing heritability, as expected. However, the increase in accuracy of total EBV of MABLUP methods in comparison to CONBLUP was largest with a low heritability. For ungenotyped animals, the increase in accuracy with MABLUP in comparison to CONBLUP was smaller, e.g. from 0.35 to 0.37 with HAP4 at a heritability of 0.03 , but the increase in accuracy was negligible when the herita- bility was 0.30 . HAP4 had in all cases the highest accuracies for QTL-EBV, polygenic EBV and total EBV, i.e. the ranking of the methods did not change.

Table 7 shows the regression of true on estimated breeding values for different values of heritability for the three MABLUP methods when the marker distance was $0.1 \mathrm{cM}$ for genotyped males and ungenotyped females. The regressions for QTL-EBV were substantially lower 


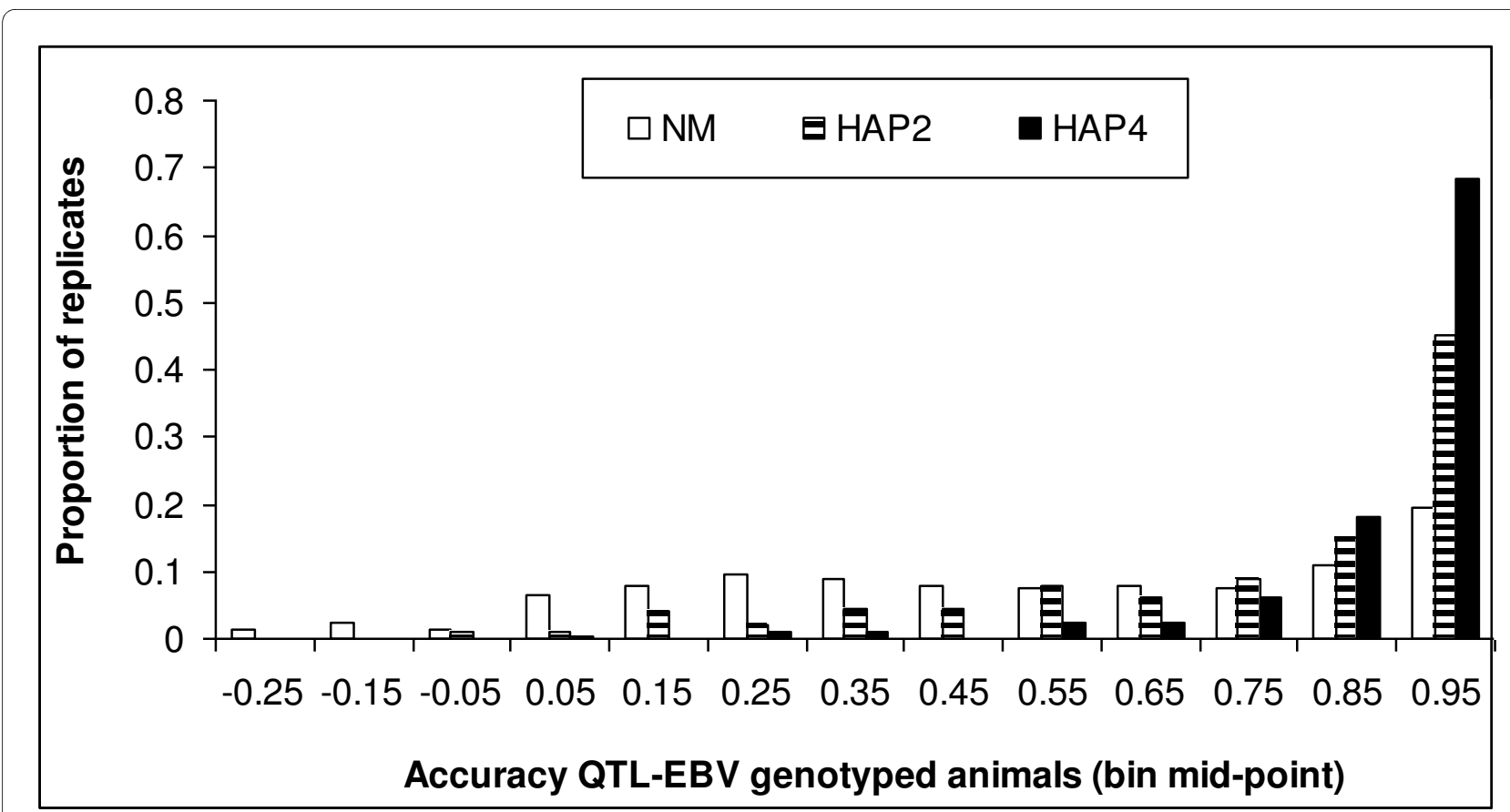

Figure 4 Frequency distribution of accuracy of QTL-EBV of genotyped animals. Proportion of replicates per 0.1-bin-class for accuracy of QTL-EBV of genotyped animals for neighboring marker (NM), 2-marker haplotype (HAP2) and 4-marker haplotype (HAP4); sires and males in last generation are genotyped, distance between markers is $0.1 \mathrm{cM}$, heritability is 0.3 , the QTL explains 15\% of the genetic variance, average of 200 replicates.

Table 6: Accuracies of QTL-EBV, polygenic EBV and total EBV for genotyped males and ungenotyped females

\begin{tabular}{|c|c|c|c|c|c|c|c|c|}
\hline \multirow[b]{2}{*}{ EBV } & \multirow[b]{2}{*}{$h^{2}$} & \multirow[b]{2}{*}{ CONBLUP } & \multicolumn{3}{|c|}{ Genotyped } & \multicolumn{3}{|c|}{ Ungenotyped } \\
\hline & & & NM & HAP2 & HAP4 & NM & HAP2 & HAP4 \\
\hline \multirow[t]{3}{*}{ QTL } & 0.03 & & 0.568 & 0.723 & 0.796 & 0.371 & 0.475 & 0.524 \\
\hline & 0.10 & & 0.542 & 0.770 & 0.865 & 0.349 & 0.493 & 0.554 \\
\hline & 0.30 & & 0.534 & 0.775 & 0.912 & 0.336 & 0.491 & 0.580 \\
\hline \multirow[t]{3}{*}{ Polygenic } & 0.03 & & 0.333 & 0.336 & 0.336 & 0.335 & 0.339 & 0.339 \\
\hline & 0.10 & & 0.444 & 0.452 & 0.456 & 0.454 & 0.444 & 0.452 \\
\hline & 0.30 & & 0.567 & 0.576 & 0.583 & 0.566 & 0.575 & 0.582 \\
\hline \multirow[t]{2}{*}{ Total } & 0.03 & 0.351 & 0.387 & 0.407 & 0.418 & 0.362 & 0.368 & 0.371 \\
\hline & 0.30 & 0.594 & 0.605 & 0.616 & 0.622 & 0.595 & 0.596 & 0.596 \\
\hline
\end{tabular}

Accuracies ${ }^{1}$ of QTL-EBV, polygenic EBV and total EBV for different values of heritability for marker-assisted BLUP with neighboring marker (NM), 2-marker haplotype (HAP2) and 4-marker haplotypes (HAP4) and conventional BLUP (CONBLUP) (sires and males in last generation are genotyped; distance between markers is $0.1 \mathrm{cM}$, the QTL explains $15 \%$ of the genetic variance, results are averages of 200 replicates) 1Standard errors were between 0.007 and 0.022 for QTL-EBV, between 0.002 and 0.006 for polygenic EBV and between 0.002 and 0.005 for total EBV 
Table 7: Regression coefficients of estimated breeding values for genotyped males and ungenotyped females

\begin{tabular}{|c|c|c|c|c|c|c|c|c|}
\hline \multirow[b]{2}{*}{ EBV } & \multirow[b]{2}{*}{$h^{2}$} & \multirow[b]{2}{*}{ CONBLUP } & \multicolumn{3}{|c|}{ Genotyped } & \multicolumn{3}{|c|}{ Ungenotyped } \\
\hline & & & NM & HAP2 & HAP4 & NM & HAP2 & HAP4 \\
\hline \multirow[t]{3}{*}{ QTL } & 0.03 & & 0.867 & 1.115 & 1.143 & 0.797 & 1.109 & 1.165 \\
\hline & 0.10 & & 0.772 & 0.899 & 0.955 & 0.809 & 0.889 & 0.953 \\
\hline & 0.30 & & 0.869 & 0.909 & 0.917 & 0.744 & 0.884 & 0.910 \\
\hline \multirow[t]{3}{*}{ Polygenic } & 0.03 & & 0.945 & 0.962 & 0.970 & 0.948 & 0.965 & 0.975 \\
\hline & 0.10 & & 0.950 & 0.973 & 0.985 & 0.951 & 0.973 & 0.985 \\
\hline & 0.30 & & 0.951 & 0.966 & 0.976 & 0.954 & 0.965 & 0.973 \\
\hline \multirow[t]{3}{*}{ Total } & 0.03 & 0.972 & 0.954 & 0.991 & 0.986 & 0.989 & 0.997 & 0.989 \\
\hline & 0.10 & 0.987 & 0.981 & 0.975 & 0.974 & 1.022 & 1.014 & 1.011 \\
\hline & 0.30 & 0.966 & 1.000 & 0.988 & 0.979 & 1.032 & 1.029 & 1.026 \\
\hline
\end{tabular}

Regression ${ }^{1}$ of true on estimated breeding values for QTL-EBV, polygenic EBV and total EBV for genotyped males and ungenotyped females for different values of heritability for marker-assisted BLUP with neighboring marker (NM), 2-marker haplotype (HAP2) and 4-marker haplotypes (HAP4) and conventional BLUP (CONBLUP) (sires and males in last generation are genotyped; distance between markers is $0.1 \mathrm{cM}$, the QTL explains $15 \%$ of the genetic variance, results are averages of 200 replicates)

1Standard errors were between 0.015 and 0.060 for QTL_EBV, between 0.004 and 0.015 for polygenic EBV and between 0.004 and 0.014 for total EBV; regressions where the variance of the predicted $n h c$ was smaller than 0.0001 were omitted (denominator of regression coefficient)

Table 8: Bias in estimated breeding values for genotyped males and ungenotyped females

\begin{tabular}{|c|c|c|c|c|c|c|c|c|}
\hline \multirow[b]{2}{*}{ EBV } & \multirow[b]{2}{*}{$h^{2}$} & \multirow[b]{2}{*}{ CONBLUP } & \multicolumn{3}{|c|}{ Genotyped } & \multicolumn{3}{|c|}{ Ungenotyped } \\
\hline & & & NM & HAP2 & HAP4 & NM & HAP2 & HAP4 \\
\hline \multirow[t]{3}{*}{ QTL } & 0.03 & & -0.008 & -0.007 & -0.001 & -0.009 & -0.007 & -0.001 \\
\hline & 0.10 & & -0.022 & -0.013 & 0.000 & -0.023 & -0.014 & -0.002 \\
\hline & 0.30 & & -0.057 & -0.028 & 0.008 & -0.060 & -0.032 & 0.003 \\
\hline \multirow[t]{3}{*}{ Polygenic } & 0.03 & & 0.006 & 0.002 & -0.003 & 0.006 & 0.001 & -0.003 \\
\hline & 0.10 & & 0.064 & 0.049 & 0.035 & 0.064 & 0.049 & 0.035 \\
\hline & 0.30 & & 0.125 & 0.086 & 0.053 & 0.126 & 0.087 & 0.055 \\
\hline \multirow[t]{3}{*}{ Total } & 0.03 & 0.007 & -0.002 & -0.005 & -0.004 & -0.003 & -0.006 & -0.005 \\
\hline & 0.10 & 0.042 & 0.041 & 0.035 & 0.034 & 0.041 & 0.035 & 0.034 \\
\hline & 0.30 & 0.036 & 0.068 & 0.058 & 0.061 & 0.067 & 0.055 & 0.058 \\
\hline
\end{tabular}

Bias $^{1}$ (estimated - true breeding value) in QTL-EBV, polygenic EBV and total EBV for genotyped males and ungenotyped females for different values of heritability for marker-assisted BLUP with neighboring marker (NM), 2-marker haplotype (HAP2) and 4-marker haplotypes (HAP4) and conventional BLUP (CONBLUP) (sires and males in last generation are genotyped; distance between markers is $0.1 \mathrm{cM}$, the QTL explains $15 \%$ of the genetic variance, results are averages of 200 replicates)

'Standard errors were between 0.003 and 0.012 for $h^{2}=0.03$, between 0.005 and 0.025 for $h^{2}=0.10$ and between 0.009 and 0.037 for $h^{2}=0.30$ 
than 1.0 in the majority of the situations, except when the heritability was 0.03 . This indicated that the variance of the QTL-effect was overestimated when the heritability was 0.10 and 0.30 . HAP4 had regression coefficients closest to 1.0 indicating that in this case, overestimation was the smallest. Regressions for polygenic and total EBV were in most cases close to one. The variances of the polygenic EBV were slightly overestimated in all cases. The variances of the total EBV were slightly overestimated for genotyped males for CONBLUP and MABLUP and slightly underestimated for ungenotyped females with MABLUP, but overestimated with CONBLUP. Overall, the variance of total EBV was less biased with MABLUP than with CONBLUP.

Table 8 shows the bias in estimated breeding values for different values of heritability using the three MABLUP methods and CONBLUP for genotyped males and ungenotyped females when the marker distance was 0.1 cM. The polygenic EBV were on average biased upwards and the QTL-EBV were biased downwards, or in other words the QTL-effects were underestimated, but the polygenic EBV absorbed this effect. The total EBV were biased upwards for all methods when the heritability was 0.10 and 0.30 , due to the shift of the estimated mean in the model, which was caused by genetic trend due to selection and the change in allele frequency of the QTL. Bias was largest for NM, whereas HAP2 and HAP4 were similar. Without selection total EBV were unbiased (results not shown). There was hardly any difference in bias between genotyped males and ungenotyped females. Adding the overall mean to the EBV removed the bias in total EBV. It can be concluded that total EBV of MABLUP and EBV of CONBLUP were biased due to selection, but this bias did no affect the ranking of animals.

\section{Discussion}

In this study we developed a method to predict haplotypes of ungenotyped animals using pedigree information of genotyped animals in mixed model equations and we evaluated the use of these predicted haplotypes in marker-assisted BLUP. The method is an extension of Gengler et al. $[14,15]$ to multi-allelic markers or haplotypes. The method was evaluated with Monte Carlo simulation. Clearly the predicted number of haplotype copies was regressed towards the mean and more so than the gene contents in Gengler et al. [14,15], especially when the frequency of a certain haplotype was low, which is more likely with longer haplotypes because of an increasing number of haplotypes. When using only a neighbor marker, the predicted gene contents were in the same range as in Gengler et al. $[14,15]$. Because of the almostunity heritability the number of haplotype copies is hardly regressed towards the mean for genotyped animals. The accuracy of the predicted haplotypes was lower for HAP4 than for HAP2 and decreased with increasing marker distance due to the increased probability of recombination. Lowering the heritability might be an option, taking into account that the number of haplotype copies from parent to offspring is not fully heritable but subject to recombination. However, BLUP is very robust against changes in heritability and preliminary results showed no effect when the heritability was changed to 0.95 .

The 4-marker haplotype gave the best results in marker-assisted breeding value estimation. It captured 90\% of the QTL-variance when markers were separated by $0.1 \mathrm{cM}$. Because of this high proportion of explained QTL-variance, the proportion of QTL-variance explained by the haplotype can not increase much, and therefore we did not consider longer haplotypes. Furthermore, longer haplotypes are more subject to recombination, decreasing the accuracy of predicted number of haplotype copies. Hayes et al. [6] found that 6-marker haplotypes explained more QTL-variance than 4-marker haplotypes, but had much lower proportions of QTL-variance explained by the markers due to lower marker density and lower LD. Hayes et al. [6] found that the increase in accuracy was much higher with haplotypes than with using a neighbor marker in agreement with this study. Calus et al. [22] investigated the use of different definitions of haplotypes on the accuracy of genomic selection and found that with a high marker density the regression on single SNP worked almost as well as haplotypes with two markers. In their study all SNP were used for a single SNP regression, whereas in this study only one SNP was used to estimate the QTL-effect. This disfavored the neighbor marker method in our study, although the ranking of the alternatives is the same as in Calus et al. [22]. In the context of QTL fine-mapping, Grapes et al. [23] found that single marker regression with 10 markers performed worse than an IBD-method using linkage disequilibrium and linkage analysis information with a haplotype window of 10 markers, but single marker regression performed similarly when 20 markers were used. Zhao et al. [24] found that the power of a model with regression on two or four SNP yielded higher power to detect QTL than 2- or 4-marker haplotypes. This suggests that ranking of methods for QTL mapping might be different than for accuracy of marker-assisted or genomic selection [25].

The proportion QTL-variance explained by the haplotypes or the neighbor marker $\left(r^{2}\right)$ was higher than in Hayes et al. [6]. At marker distances ranging from 0.1 to $1.0 \mathrm{cM}$, estimated $r^{2}$ in cattle populations have been found lower $(\sim 0.05-0.27)$ than those found in this simulation study [6,26-28]. However, in pig and poultry populations higher $r^{2}$ have been estimated $(\sim 0.20-0.50$ in pigs and poultry) $[29,30]$, resembling the observed $r^{2}$ in our study. The $r^{2}$ between neighbor marker and QTL or 
between pairs of markers followed the expected $r^{2}$ based on distance in $\mathrm{cM}$ and the effective population size [31]. The lower $r^{2}$ values found at short distance in cattle populations is probably due to much higher effective population sizes in the past, because LD at short distances reflects more the past effective population size [32]. As a consequence of lower LD at short distances in cattle, a higher SNP density than that used in this study is necessary to achieve in cattle the same accuracy of QTL-EBV as presented here.

Haplotypes were assumed to be unrelated in this study and it was assumed that the same QTL-allele is linked to a certain haplotype (identity-by-state = IBS). Due to recombination, linkage phases between haplotypes and QTL may be different in different families. In the context of genomic selection, Calus et al. [22] compared 2marker IBS-haplotypes with 2- and 10-marker identityby-descent haplotypes using combined linkage disequilibrium linkage analysis information (LDLA) to construct the inverse IBD-matrices. They found that IBD-haplotypes yielded higher accuracies, especially when using 10marker windows, but at the cost of much higher computing time. The difference between IBS and IBD-haplotypes decreased with increasing marker density. Therefore, in our study it is unlikely that IBD-haplotypes would increase accuracy significantly when the distance between the markers is less than $0.1 \mathrm{cM}$.

A major disadvantage of using haplotypes is the need to phase the data. Hayes et al. [6] estimated the effect of haplotyping errors on the proportion of QTL-variance explained by the haplotypes in their data set and found a limited effect, but suggested that phasing errors are dependent on the data structure used. Accurate and fast algorithms are available for use in livestock populations $[33,34,28]$. Windig and Meuwissen [34] have shown that their algorithm is very fast and yields almost perfect haplotype reconstruction with dense marker maps in pedigreed populations. Its performance was similar to that of SIMWALK2 [35] in terms of accuracy, but with a much lower computing time. Furthermore, the presented method can accommodate haplotyping errors, e.g. by adjusting the heritability of $n h c$ to a lower value, albeit at the expense of a lower accuracy.

The major advantage of the method used in this study is its computing efficiency, because optimized BLUP software can be used to predict haplotypes. The computation time was respectively $\sim 4,6$ and $10 \mathrm{~s}$ for neighboring marker (NM), 2-marker haplotypes (HAP2) and 4marker haplotypes (HAP4) to predict the genotypes/haplotypes on a dual-processor 64-bit Windows PC with 2.40 $\mathrm{GHz}$ and $36 \mathrm{~GB}$ of RAM; programs were compiled for 32bit. Therefore, breeding companies do not need other software for imputing genotypes, which is usually much slower and much more memory intensive, prohibiting its use for large populations, e.g. with more than a million animals. An additional advantage is that no assumptions are needed on where ungenotyped animals should appear in the pedigree, it can handle all possible scenarios. Therefore, the proposed method is very suitable for application of marker-assisted breeding value estimation in large populations, such as national evaluations in cattle. Also for genomic selection purposes the method is very useful, e.g. for 50,000 SNP-markers it would take only about two days on a single processor to predict all SNPgenotypes or haplotypes for a similar number of animals as in this study.

The use of 4-marker haplotypes (HAP4) increased the accuracy of marker-assisted breeding value estimation substantially in comparison to conventional breeding value estimation for genotyped animals, but the benefit for ungenotyped animals was small in agreement with Mulder et al. [8]. However, with a low heritability, ungenotyped animals gained considerably in accuracy. This can be visualized by approximating the accuracy of the total EBV $\left(r_{\text {totalEBV }}\right)$ as:

$$
r_{\text {totalEBV }}=\sqrt{\left(1-q^{2}\right) r_{A_{\text {pol }}}^{2}+q^{2} r_{h}^{2}}
$$

where $q^{2}$ is the proportion of genetic variance explained by the haplotypes $\left(=r_{A_{q t}}^{2} \times Q^{2}\right.$, where $r_{A_{q t l}}$ is the accuracy of the QTL-EBV and $Q^{2}$ is the amount of genetic variance explained by the QTL), $r_{A_{p o l}}$ is the accuracy of the polygenic EBV and $r_{h}$ is the accuracy of the predicted number of haplotype copies. If we take the situation where the heritability is 0.03 , the distance between markers is $0.1 \mathrm{cM}$ and the QTL explains $15 \%$ of the genetic variance, $r_{A_{p o l}}$ is 0.34 (Table 6) and we assume that $q^{2}$ is 0.10 (assuming $r_{A_{q t l}}=0.8$ (Table 6)), then Equation (5) yields $r_{\text {totalEBV }}=0.374$, close to the value in Table 6. Using Equation (5), we can also quantify the benefit of genomewide EBV for ungenotyped animals. Lets assume that we can explain $90 \%$ of the genetic variance by markers $\left(q^{2}=\right.$ $0.9)$, then we can increase $r_{\text {totalEBV }}$ up to 0.58 assuming that $r_{A_{p o l}}$ is constant. So even for ungenotyped animals genome-wide EBV can increase accuracy in comparison to conventional BLUP, especially for low heritability traits, when their paternal ancestors are genotyped. 


\section{Conclusions}

In this study we show that mixed model equations can be used to predict number of haplotype copies for ungenotyped animals and these predicted number of haplotype copies can be used in marker-assisted breeding value estimation. Four-marker haplotypes give the highest accuracy for total estimated breeding values. The accuracy of the total EBV increases for genotyped animals, but for ungenotyped animals the increase is marginal unless the heritability is smaller than 0.1 . The method works best when the distance between the markers is less than $1 \mathrm{cM}$. The proposed method is computationally very efficient and suitable to apply for marker-assisted breeding value estimation in large livestock populations including effects of a number of known QTL. Marker-assisted breeding value estimation using predicted haplotypes increases accuracy especially for traits with low heritability. It is expected that genomic selection for ungenotyped animals using predicted haplotypes or marker genotypes will be beneficial especially for low heritable traits.

\section{Appendix}

\section{Derivation of haplotype variance used in mixed models}

Assuming that the haplotypes explain $100 \%$ of the QTLvariance, the variance of haplotype effects $\sigma_{h}^{2}$ used in Equation (4) can be calculated similarly to the variance when regressing on one bi-allelic marker/QTL:

$$
\begin{aligned}
& 2 p(1-p) \sigma_{h}^{2}=\sigma_{A_{q t l}}^{2}=2 p(1-p) \alpha^{2} \\
& \sigma_{h}^{2}=\alpha^{2}
\end{aligned}
$$

where $\alpha$ is the allele substitution effect, $p$ is the allele frequency of one of the two SNP-alleles. Extrapolating the result of Equation (A1) to $n$ haplotypes yields:

$$
\sigma_{h}^{2}=\frac{2 p(1-p) \alpha^{2}}{\sum_{i=1}^{i=n} 2 m_{i}\left(1-m_{i}\right)}
$$

where $m_{i}$ is the frequency of haplotype $i$. Assuming equal frequencies of all $n$ haplotypes yields:

$$
\sigma_{h}^{2}=\frac{2 p(1-p) \alpha^{2}}{n^{*} 2 * \frac{1}{n} * \frac{n-1}{n}}=\frac{n}{n-1} p(1-p) \alpha^{2} .
$$

The limit of Equation (A3) is:

$$
\lim _{n \rightarrow \infty} \sigma_{h}^{2}=\lim _{n \rightarrow \infty} \frac{n}{n-1} p(1-p) \alpha^{2}=0.5 \sigma_{A_{q t l}}^{2}
$$

showing that the variance of haplotype $i$ is half the additive genetic variance of the QTL with an infinite number of haplotypes. Although the result in Equation (A2) depends on haplotype frequencies and number of haplotypes, preliminary analyses showed that using the result of Equation (A4) yields high accuracies of QTLEBV. Furthermore, these preliminary analyses showed that the accuracy of the QTL-EBV is insensitive to $\sigma_{h}^{2}$.

\section{Competing interests}

The authors declare that they have no competing interests.

\section{Authors' contributions}

HAM developed the method, ran the simulations and evaluations and drafted the manuscript. MPLC and RFV discussed the method and results and helped to draft the manuscript. All authors read and approved the final manuscript.

\section{Acknowledgements}

Egbert Knol, Dieuwke Roelofs-Prins, Marc Rutten, Chris Schrooten, Addie Vereijken, Martin Lidauer, Ismo Stranden and Robin Thompson are thanked for helpful discussions about this study. We would like to thank two anonymous reviewers for giving constructive suggestions for improving the manuscript. The work was financially supported by CRV, Hendrix Genetics, IPG and the European Commission, within the 6th Framework project SABRE, contract No. FOOD-CT-2006-016250. The text represents the authors' views and does not necessarily represent a position of the Commission who will not be liable for the use made of such information.

\section{Author Details}

Animal Breeding and Genomics Centre, Wageningen UR Livestock Research, PO Box 65, 8200 AB Lelystad, The Netherlands

Received: 21 October 2009 Accepted: 22 March 2010

Published: 22 March 2010

\section{References}

1. Dekkers JCM: Commercial application of marker- and gene-assisted selection in livestock: Strategies and lessons. J Anim Sci 2004, 82(E Suppl):E313-E328

2. Grisart N, Coppieters W, Farnir F, Karim L, Ford C, Berzi P, Cambisamo N, Mni MRS, Simon P, Spelman R, Georges M, Snell R: Positional candidate cloning of a QTL in dairy cattle: identification of a missense mutation in the bovine DGAT1 gene with major effect on milk yield and composition. Genome Res 2002, 12:222-231.

3. Winter A, Kramer W, Werner FAO, Kollers S, Kata S, Durstewitz G, Buitkamp J, Womack JE, Thaller G, Fries R: Association of a lysine-232/alanine polymorphism in a bovine gene encoding acyl-CoA:diacylglycerol acyltransferase (DGAT1) with variation at a quantitative trait locus for milk fat content. Proc Nat Acad Sci USA 2002, 99:9300-9305.

4. Van Laere A-S, Nguyen M, Braunschweig M, Nezer C, Collete C, Moreau L, Archibald AL, Haley CS, Buys N, Tally M, Andersson G, Georges M, Andersson L: A regulatory mutation in IGF2 causes a major QTL effect on muscle growth in pigs. Nature 2003, 425:832-836.

5. Villanueva B, Pong-Wong R, Woolliams JA: Marker assisted selection with optimised contributions of the candidates to selection. Genet Sel Evol 2002, 34:679-703.

6. Hayes BJ, Chamberlain AJ, McPartlan H, Macleod I, Sethuraman L, Goddard ME: Accuracy of marker-assisted selection with single markers and marker haplotypes in cattle. Genet Res Camb 2007, 89:215-220. 
7. Fernando RL, Grossman M: Marker assisted selection using best linear unbiased prediction. Genet Sel Evol 1989, 21:467-477.

8. Mulder HA, Meuwissen THE, Calus MPL, Veerkamp RF: The effect of missing marker genotypes on the accuracy of gene-assisted breeding value estimation: a comparison of methods. Animal 2010, 4:9-19.

9. Meuwissen THE: Determining haplotypes and IBD-probabilities from dense-marker genotypes in large complex pedigrees. Proceedings 8 th World Congress Genetics Applied to Livestock Production; Belo Horizonte, Brazil. Communication 2006:20-12.

10. Van Arendonk JAM, Smith C, Kennedy BW: Method to estimate genotype probabilities at individual loci in farm livestock. Theor App/ Genet 1989, 78:735-740

11. Fernando RL, Stricker C, Elston RC: An efficient algorithm to compute the posterior genotypic distribution for every member of a pedigree without loops. Theor Appl Genet 1993, 87:89-93.

12. Thallman RM, Bennett GL, Keele JW, Kappes SM: Efficient computation of genotype probabilities for loci with many alleles: I. Allelic peeling. $J$ Anim Sci 2001, 79:26-33.

13. Thallman RM, Bennett GL, Keele JW, Kappes SM: Efficient computation of genotype probabilities for loci with many alleles: II. Iterative method for large, complex pedigrees. J Anim Sci 2001, 79:34-44.

14. Gengler N, Mayeres P, Szydlowski M: A simple method to approximate gene content in large pedigree populations: application to the myostatin gene in dual-purpose Belgian Blue cattle. Animal 2007 1:21-27.

15. Gengler N, Abras S, Verkenne C, Vanderick S, Szydlowski M, Renaville R: Accuracy of prediction of gene content in large animal populations and its use for candidate gene detection and genetic evaluation. $J$ Dairy Sci 2008, 91:1652-1659.

16. Haldane JBS: The combination of linkage values and the calculation of distances between the loci of linked factors. J Genet 1919, 8:299-309.

17. Bulmer MG: The effect of selection on genetic variability. Am Nat 1971, 105:201-211.

18. Meuwissen THE, Luo Z: Computing inbreeding coefficients in large populations. Genet Sel Evol 1992, 24:305-313.

19. Lidauer M, Stranden I: Fast and flexible program for genetic evaluation in dairy cattle. Interbull Bull 1999, 20:20-25.

20. Hill WG, Robertson A: Linkage disequilibrium in finite populations. Theor App/ Genet 1968, 38:226-231.

21. Zhao H, Nettleton D, Soller M, Dekkers JCM: Evaluation of linkage disequilibrium measures between multi-allelic markers as predictors of linkage disequilibrium between markers and QTL. Genet Res Camb 2005, 86:77-87.

22. Calus MPL, Meuwissen THE, De Roos APW, Veerkamp RF: Accuracy of genomic selection using different methods to define haplotypes. Genetics 2008, 178:553-561.

23. Grapes L, Dekkers JCM, Rothschild MF, Fernando RL: Comparing linkage disequilibrium-based methods for fine mapping quantitative trait loci. Genetics 2004, 166:1561-1570

24. Zhao HH, Fernando RL, Dekkers JCM: Power and precision of alternate methods for linkage disequilibrium mapping of quantitative trait loci. Genetics 2007, 175:1975-1986.

25. Calus MPL, Meuwissen THE, Windig JJ, Knol EF, Schrooten C, Vereijken ALJ, Veerkamp RF: Effects of the number of markers per haplotype and clustering of haplotypes on the accuracy of QTL mapping and prediction of genomic breeding values. Genet Sel Evol 2009, 41:11

26. De Roos APW, Hayes B, Spelman R, Goddard ME: Linkage disequilibrium and persistence of phase in Holstein-Friesian, Jersey and Angus cattle. Genetics 2008, 179:1503-1512.

27. Khatkar MS, Nicholas FW, Collins AR, Zenger KR, Cavanagh JAL, Barris W, Schnabel RD, Taylor JF, Raadsma HW: Extent of genome-wide linkage disequilibrium in Australian Holstein-Friesian cattle based on a highdensity SNP panel. BMC Genomics 2008, 9:187-194.

28. Sargolzaei M, Schenkel FS, Jansen GB, Schaeffer LR: Extent of linkage disequilibrium in Holstein cattle in North America. J Dairy Sci 2008, 91:2106-2117

29. Du F-X, Clutter AC, Lohuis MM: Characterizing linkage disequilibirum in pig populations. Int J Biol Sci 2007, 3:166-178.

30. Andreescu C, Avendano S, Brown SR, Hassen A, Lamont SJ, Dekkers JCM: Linkage disequilibrium in related breeding lines of chickens. Genetics 2007, 177:2161-2169.
31. Sved JA: Linkage disequilibrium and homozygosity of chromosome segments in finite populations. Theor Pop Biol 1971, 2:125-141.

32. Hayes BJ, Visscher PM, McPartlan HC, Goddard ME: Novel multilocus measure of linkage disequilibrium to estimate past effective population size. Genome Res 2003, 13:635-643.

33. Scheet $P$, Stephens M: A fast and flexible statistical model for large-scale population genotype data: applications to inferring missing genotypes and haplotypic phase. Am J Hum Genet 2006, 78:629-644

34. Windig JJ, Meuwissen THE: Rapid haplotype reconstruction in pedigrees with dense marker maps. J Anim Breed Genet 2004, 121:26-39.

35. Sobel $E$, Lange $K$ : Descent graphs in pedigree analysis: applications to haplotyping, location scores, and marker-sharing statistics. Am J Hum Genet 1996, 58:1323-1337.

doi: $10.1186 / 1297-9686-42-10$

Cite this article as: Mulder et al., Prediction of haplotypes for ungenotyped animals and its effect on marker-assisted breeding value estimation Genetics Selection Evolution 2010, 42:10

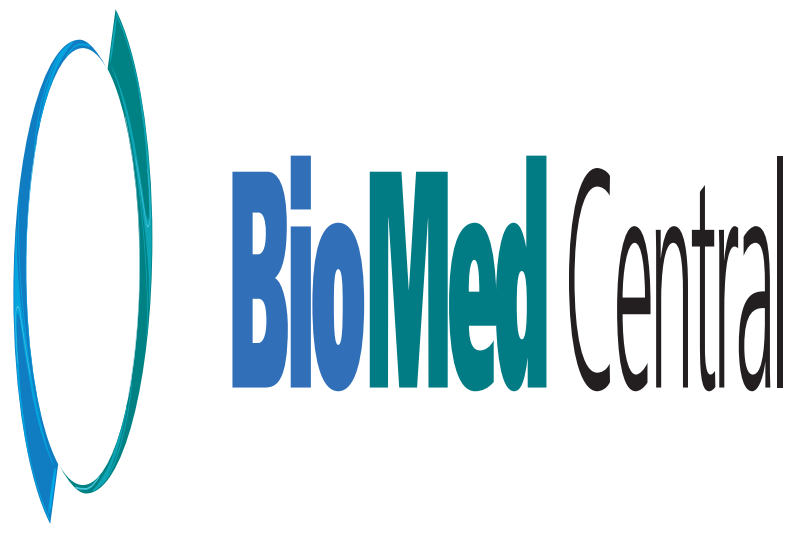

